\title{
An uncommon cause of common presentation - Gall stone ileus: A case report and review of literature
}

\author{
Anand Munghate', Sushil Mittal ${ }^{2}$, Harnam Singh ${ }^{3}$, Anjna Garg ${ }^{4}$, Gurpreet Singh ${ }^{5}$, Jyoti Sharma 6 \\ ${ }^{1}$ Junior Resident, ${ }^{2}$ Associate Professor, ${ }^{3}$ Assistant Professor, ${ }^{4}$ Senior Resident, ${ }^{5}$ Junior Resident, Department of General Surgery, Government \\ Medical College, Patiala 147001, India, ${ }^{6}$ Junior Resident, Department of Pathology, Pandit Bhagwan Dayal Sharma Post Graduate Institute \\ of Medical Sciences, Rohtak 124001, India
}

\section{A B S TR A C T}

About $1 \%-4 \%$ of all cases of mechanical intestinal obstruction were caused by rare disease gallstone ileus. It is an uncommon complication of cholelithiasis. The formation of a fistula between the gallbladder and the duodenum may allow a gallstone to enter the intestinal tract. Gallstone ileus usually occurs in the elderly with a female predominance and is associated with a high mortality rate. The diagnosis of gallstone ileus entails a clinical challenge, especially in older patients in whom it may be easily overlooked. Here we report a case of 75 year old female patient presented in emergency with 4 day history of nausea, vomiting and pain in epigastric region. Emergent laparotomy was done and it was surprise to see that the cause of obstruction was gall stone ileus, enterolithotomy and repair was done.

Key words: Gallstone ileus, Intestinal obstruction, Enterolithotomy
Access this article online

Website:

http://nepjol.info/index.php/AJMS

\section{INTRODUCTION}

Adhesions, congenital abnormalities, intussusception, volvulus, foreign bodies, gallstones, bezoars and tumors are the most common cause of the mechanical small bowel intestinal obstruction. ${ }^{1}$ Gallstone-induced intestinal obstruction, also referred to as gallstone ileus, is an uncommon and potentially serious complication of cholelithiasis. It occurs when gallstones migrate from the gallbladder to the bowel through a cholecystoenteric fistula, causing a mechanical obstruction. Cholecystoduodenal fistulas are the most frequent (75\%), followed by cholecystocolic fistulas (10-20\%), and a variety of other types (15\%). Spontaneous enterobiliary fistulas occur secondary to biliary disease and disease of adjacent structures. ${ }^{2}$ Gallstone ileus accounts for $1-4 \%$ of all cases of surgery for intestinal obstruction. However, in the over-65-year age group, the prevalence is much higher with female to male ratio as 3.5-6.0:1, with gallstone ileus accounting for $25 \%$ of all cases of nonstrangulated small bowel obstruction. $^{3}$
Here we report a case of gallstone ileus with two biliary stones causing intestinal obstruction with review of literature of this uncommon disease.

\section{CASE REPORT}

A 75 year old female patient presented in emergency department with 4 day history of nausea, vomiting and pain in epigastric region. Abdominal examination was revealed distended abdomen with tenderness in epigastric region. Her bowel sounds were diminished. Routine blood examinations showed leucocytosis. Her chest X-ray was normal while abdominal X-ray showed distended gut loops with significant air fluid level. On the basis of findings, decision was made to operate. At laparotomy through midline incision, careful exploration and palpation of small bowel revealed a large impacted gallstone in the mid of ileum causing an intestinal obstruction (Figure 1). Proximally to obstruction a small gall stone was also present. A simple enterolithotomy was done for impacted gallstone (Figure 2). On further exploration of abdominal cavity a cholecystoduodenal 
fistula was identified between gallbladder wall and duodenal loops (Figure 3). Patient recovered well and dischared on 12 th postoperative day.

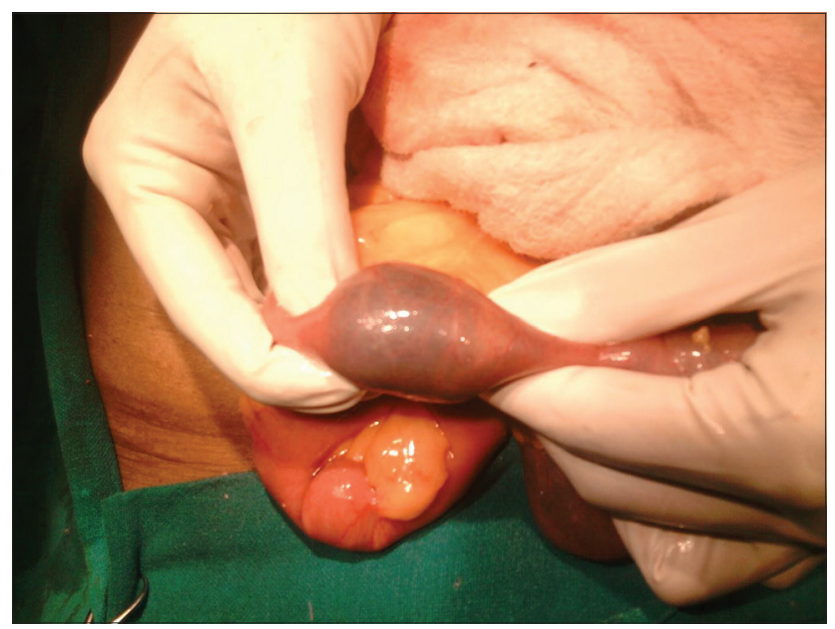

Figure 1: A large impacted gallstone in the mid of ileum causing an intestinal obstruction

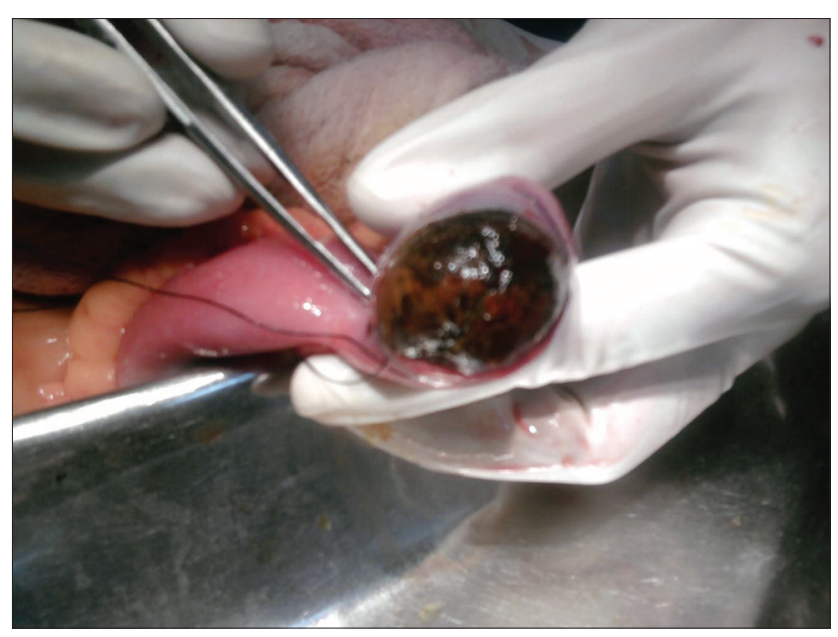

Figure 2 : A simple enterolithotomy for impacted gallstone

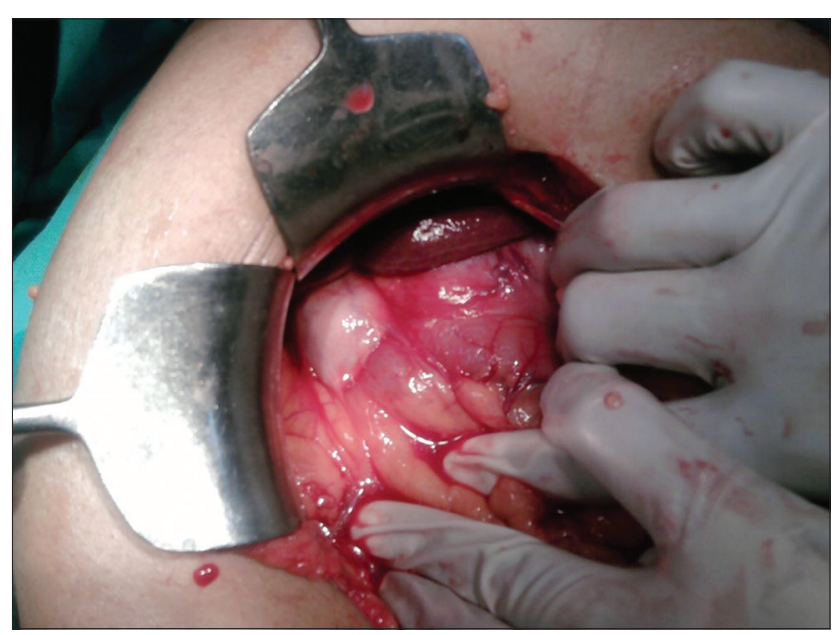

Figure 3: A cholecystoduodenal fistula between gallbladder wall and duodenal loops

\section{DISCUSSION}

Gallstone is a common disease which is symptomatic only in $20 \%-30 \%$ of patients with biliary colic being the most common symptom. ${ }^{4}$ Acute cholecystitis, acute pancreatitis, choledocholithiasis with or without cholangitis, and a gangrenous gallbladder are the most common complications of gallstone disease while Mirizzi syndrome, cholecystocholedochal fistula and gallstone ileus are uncommon complication. ${ }^{3,4}$

Biliary-enteric fistula is the major pathologic mechanism of gallstone ileus. It is generally believed that pericholecystic inflammation after cholecystitis, as well as pressure necrosis by the gallstone against the biliary wall, may lead to formation of a biliary enteric fistula. Fistula formation is a complication of $2 \%$ to $3 \%$ of all cases of cholelithiasis with associated episodes of cholecystitis. ${ }^{5}$ In a large series, $89.5 \%$ of patients with cholecystoenteric fistulae were also found to have a common bile duct obstruction caused by an extrinsic compression from an impacted stone in the cystic duct, known as Mirizzi syndrome. A biliary enteric fistula provides a passage for large gallstones to enter the bowel and eventually cause gallstone ileus. ${ }^{6}$ To date, the largest review of reported cases of gallstone ileus, which consisted of 1001 cases, was published by Reisner and Cohen in 1994 who reported that the most common locations of impaction of gallstone are the terminal ileum and the ileocecal valve because of the anatomical small diameter and less active peristalsis. They also found that the less common locations for impaction are the jejunum, the ligament of Treitz, and the stomach, while the duodenum and colon are the rare locations for impaction. ${ }^{3}$

The gallstone ileus is most commonly characterised by the like nausea, vomiting and epigastric pain. Moreover, a small portion of patients may present with hematemesis secondary to duodenal erosions. The diagnosis of gallstone ileus is difficult, usually depending on the radiographic findings. In $50 \%$ of cases the diagnosis is often only made at laparotomy. ${ }^{3}$ Lassandro et $\mathrm{al}^{7}$ compared the clinical value of plain abdominal film, abdominal ultrasound and abdominal CT in diagnosing 27 cases of gallstone ileus, and found that the Rigler's triad (mechanical small bowel obstruction, ectopic gallstone, and air in the biliary tree on abdominal x-ray film) presents $14.81 \%$ in plain abdominal film, $11.11 \%$ in abdominal US, and $77.78 \%$ in abdominal CT, respectively. The abdominal CT offers crucial evidence not only for the diagnosis of gallstone ileus but also to opt for appropriate management strategies.

The primary goal of treatment in gallstone ileus is early relief of intestinal obstruction and minimization of morbidity and mortality. The debate is still going on 
about the choice of procedure, whether a one stage or two-stage surgical procedure or enterolithotomy alone. The one-stage procedure includes enterolithotomy, cholecystectomy, and fistula repair. The two stage procedure includes initial urgent enterolithotomy followed 4-6 weeks later by cholecystectomy and fistula closure. However, enterolithotomy alone is increasingly being offered to patients with gallstone ileus in an emergency setting, without follow-up definitive biliary tract surgery. ${ }^{8}$ As in our case we did not perform the second operation because of patient denial.

Tan et $\mathrm{al}^{9}$ compared the two surgical strategies; enterolithotomy alone and enterolithotomy with cholecystectomy for the emergent treatment of gallstone ileus and concluded both the procedures as safe with no mortality- however, the better was enterolithotomy. In their combined series, Reisner and Cohen ${ }^{3}$ published operative mortality rates of $16.9 \%$ for one-stage repair and $11.7 \%$ for enterolithotomy $(\mathrm{p}<0.17)$.

Recently, laparoscopy-guided enterolithotomy has become the preferred surgical approach in treating gallstone ileus. The non-surgical treatment of gallstone ileus has been suggested, including endoscopic removal and shockwave lithotripsy depending upon the location of obstruction. The postoperative recurrence rate of gallstone ileus is $4.7 \%$ and only $10 \%$ of patients require secondary biliary surgery for recurrent biliary symptoms., ${ }^{3,10}$

\section{CONCLUSION}

In a patient with history of cholelithiasis with nausea, vomiting and abdominal pain, gallstone ileus must be considered as a differential diagnosis. Abdominal CT is usually the preferred diagnostic modality because of its rapid diagnosis and high resolution. Although there are no strong evidences in favor of enterolithotomytomy alone or one-stage or two stage repair. Our results suggest that enterolithotomytomy is safe procedure for gallstone ileus and not associated with increased long-term biliary complications.

\section{REFERENCES}

1. Intestinal obstruction. (2010, September 18). Mayo Clinic. Retrieved April 27, 2014, from http://www.mayoclinic. com/health/intestinal-obstruction/DS00823.

2. Hernandez C, Heuman D and Vlahcevid ZR. Pathophysiology of disease associated with deficiency of bile acids. Principles and Practice of Gastroenterology and Hepatology. New York: Elsevier Science. 1988; 384-395.

3. Reisner RM and Cohen JR. Gallstone ileus: A review of 1001 reported cases. Am Surg. 1994; 60:441-446.

4. Berger MY, van der Velden JJ, Lijmer JG, de Kort H, Prins A and Bohnen AM. Abdominal symptoms: do they predict gallstones? A systematic review. Scand J Gastroenterol 2000; 35: 70-76.

5. Giese A, Zieren J, Winnekendonk $G$ and Henning BF. Development of a duodenal gallstone ileus with gastric outlet obstruction (Bouveret syndrome) four months after successful treatment of symptomatic gallstone disease with cholecystitis and cholangitis: a case report J Med Case Rep 2010; 4:376.

6. Beltran M, Csendes A and Cruces K: The relationship of Mirizzi syndrome and cholecystoenteric fistula: validation of a modified classification. World J Surg 2008, 32:2237-2243.

7. Lassandro F, Gagliardi N, Scuderi M, Pinto A, Gatta G and Mazzeo R. Gallstone ileus analysis of radiological findings in 27 patients. Eur J Radiol 2004; 50: 23-29.

8. Ayantunde AA and Agrawal A. Gallstone ileus: Diagnosis and management. World J Surg 2007; 31:1292-1297.

9. Tan YM, Wong WK and Ooi LL. A comparison of two surgical strategies for the emergency treatment of gallstone ileus. Singapore Med J 2004; 45: 69-72.

10. Chou JW, Hsu CH, Liao KF, Lai HC, Cheng KS, Peng CY, et al, Gallstone ileus: Report of two cases and review of the literature, World J Gastroenterol 2007;13(8): 1295-1298.

\section{Authors Contribution:}

AM - Substantial contributions to conception and design, SM - Acquisition of data, HS - Analysis and interpretation of data, AG - Drafting the article, GS - Revising it critically for important intellectual content, JS - Final approval of the version to be published. 